\title{
Erratum to: Morphological and physiological differences between dehiscent and indehiscent anthers of Chrysanthemum morifolium
}

\author{
Jiangsong Fei ${ }^{1} \cdot$ Sue Tan $^{1} \cdot$ Fengjiao Zhang $^{1} \cdot$ Lichun Hua $^{1} \cdot$ Yuan Liao $^{1} \cdot$ \\ Weimin Fang ${ }^{1} \cdot$ Fadi Chen $^{1,2} \cdot$ Nianjun Teng ${ }^{1,2}$
}

Published online: 2 September 2016

(C) The Botanical Society of Japan and Springer Japan 2016

\section{Erratum to: J Plant Res \\ DOI 10.1007/s10265-016-0854-8}

Unfortunately, the acknowledgment was published incorrectly in the original publication of the article. The corrected version of acknowledgment is given in this erratum.
Acknowledgments This study was supported by the National Natural Science Foundation of China (31471901), the Fundamental Research Funds for the Central Universities (KYZ201308, KYTZ201602), the Natural Science Foundation of Jiangsu Province (BK20161449), and the fourth phase of Jiangsu “ 333 ” project to Dr. Nianjun Teng.

The online version of the original article can be found under doi:10.1007/s10265-016-0854-8.

Nianjun Teng

nianjunteng@163.com

Jiangsong Fei

feijiangsong@sina.com

Sue Tan

2013104119@njau.edu.cn

Fengjiao Zhang

2014204032@njau.edu.cn

Lichun Hua

2014104115@njau.edu.cn

Yuan Liao

liaoyuan@njau.edu.cn

Weimin Fang

fangwm@njau.edu.cn

Fadi Chen

fangwm@njau.edu.cn

1 College of Horticulture, Nanjing Agricultural University, Nanjing 210095, China

2 Jiangsu Province Engineering Lab for Modern Facility Agriculture Technology \& Equipment, Nanjing 210095, China 